\title{
Research Article \\ Quantitative Assessment of Microbial Contamination and Patterns of Public Behaviour with Used Toothbrushes: Implications of Storage and Replacement
}

\author{
Amreen Bashir* and Peter Lambert \\ School of Life and Health Sciences, Aston University, Birmingham, UK \\ ART ICLE INFO \\ Article history: \\ Received: 17 May, 2021 \\ Accepted: 1 June, 2021 \\ Published: 16 June, 2021 \\ Keywords: \\ Microbial contamination \\ used toothbrushes \\ toothbrush storage \\ Enterobacteria \\ Pseudomonas \\ E. coli \\ environmental

\begin{abstract}
A B S T R A C T
Introduction: Toothbrushes can readily become contaminated with microbes during use and storage. They present a potential hazard of cross-contamination and subsequent infection.

Aims: To investigate the nature and level of microbial contamination of used toothbrushes and influence of participant lifestyle.

Methods and Results: 103 used toothbrushes were donated, and microbial contents were determined by microbial culture and identification. $97 \%$ of toothbrushes revealed contamination. The microbial loads ranged from $0-10^{10} \mathrm{cfu} / \mathrm{brush}$ with median values of around $10^{7}$, with Candida isolated in the highest levels. $90 \%$ of toothbrushes were stored in bathrooms presumably exposed to environmental contamination and 72\% revealed the presence of enteric bacteria including Klebsiella, Serratia and Enterobacter. 50\% of participants kept toothbrushes for $>3$ months and $40 \%$ retained toothbrushes for 4-6 months; microbial loads were fully established during the first three months of use and did not increase further with prolonged use. Only $57 \%$ of participants brushed their teeth twice a day and $41 \%$ brushed once a day.

Conclusion: Used toothbrushes are heavily contaminated and the presence of potentially pathogenic microorganisms constitutes a potential health risk.

Significance and Impact of Study: There is an under-recognised risk of cross-contamination/infection, especially when multiple users store toothbrushes in close contact. Guidance on toothbrush age, brushing frequency and storage is not being followed. Further education is required to improve public awareness.
\end{abstract}

\section{Introduction}

Potentially pathogenic microorganisms can contaminate toothbrushes due to microbial transmission during use and from the environment. Following recommendations from dentists, the average person brushes their teeth twice daily, changing their toothbrush every 3-4 months [1, 2]. Thus, after $180+$ uses, toothbrushes may be heavily contaminated [3]. 500-700 different species of microflora have been linked to the mouth [4]. Common oral microorganisms are Candida species, Streptococcus species, Staphylococcus species and Lactobacillus species [5]. In healthy individuals, the microflora present does not cause disease. Alternatively, when microbiota balance is disrupted, the existing commensal microorganisms, and non-commensal organisms, can cause disease.
Epidemiology associated with oral microbiota include oral diseases such as periodontitis [6].

Current research has identified oral microflora contamination on used toothbrushes, and the presence of environmental pathogenic bacteria on toothbrushes of both healthy and orally diseased individuals [3, 7]. Candida sp., Corynebacterium sp., Pseudomonas sp. and coliforms have been isolated in as great as $70 \%$ of toothbrushes [8]. Similar research isolated Escherichia coli and Enterococcus species from toothbrushes [9]. Some research has investigated the effect of lifestyle variables such as alcohol consumption, smoking and diet on the oral microbiome [1012]. Despite the available research regarding the microbial transmission from mouth to the toothbrush and the identification of oral microbiome, few studies have investigated the microbial load of used toothbrushes in

*Correspondence to: Dr. Amreen Bashir, School of Life and Health Sciences, Aston University, Birmingham, UK; E-mail: a.bashir6@aston.ac.uk 
association with lifestyle and toothbrush storage, particularly within the UK. Consequently, the aim of this study was to investigate the environmental microbial contamination of used toothbrushes, comparing the impact on microbial load with lifestyle variables of toothbrush users. Our hypothesis was that environmental contamination of toothbrushes could pose a risk to users depending on the nature and level of organisms present. The study was designed to determine the identity and number of organisms obtained from toothbrushes donated from a range of healthy donors in relation to lifestyle variables. We investigated gender, age, use of toothbrush caps, use of mouthwash, alcohol consumption, smoking habits, antibiotic use, toothpaste brand, diet, frequency of toothbrush use and age of toothbrush. We did not investigate the presence of anaerobic organisms in this study since we were interested in those proliferating on the brushes under aerobic storage conditions.

\section{Study Population and Methodology}

A total of 103 used toothbrushes were donated with associated responses to a questionnaire to determine lifestyle variables relevant to toothbrush use and storage. Personal identifiable data was not collected. The Aston University Ethics Committee (application number 1572) granted ethical approval. All donated toothbrushes were placed in sterile zip-locked plastic bags by the donor and were transported to the laboratory for the microbiological examination which was carried out on the same day. Toothbrush heads were aseptically removed from handles and placed into $10 \mathrm{ml}$ of phosphate-buffered saline (PBS) containing ten $5 \mathrm{~mm}$ glass beads. This suspension was mixed by vortex for 2 minutes. Serial dilutions were performed to achieve a final concentration of $10^{-6} .100 \mu 1$ of undiluted, $10^{-2}, 10^{-4}$ and $10^{-6}$-fold dilutions were inoculated on Nutrient Agar (NA), Mannitol Salt agar (MAN), MacConkey agar (MAC), Sorbitol MacConkey agar (SMAC), Sabouraud Dextrose Agar (SAB) and Columbia Blood Agar (CBA). All plates were incubated at $37^{\circ} \mathrm{C}$ except $\mathrm{SAB}$ which was incubated at $30^{\circ} \mathrm{C}$. Presumptive plate reading was conducted at 24 hours and final counts were recorded following 48 hours incubation.

Colonies were identified using standard laboratory tests including Gram staining, catalase, oxidase, biochemical profiling as appropriate. Sterile toothbrushes were included as operational controls throughout the study. Colony counts and morphology were recorded and cfu per toothbrush was calculated. Further analysis included confirmation of the identity of Gram-negative organisms via MALDI-TOF MS in the Microbiology laboratory at Birmingham Women's and Children's NHS Foundation Trust.

\section{Statistical Analysis}

The distribution of the data for microbial loads on toothbrushes was analysed by the D'Agostino-Pearson normality test. Differences in microbial loads were investigated using the Wilcoxon matched-pairs signed-rank test for paired data samples and Mann-Whitney U-test for comparisons between two populations. All tests were conducted using GraphPad Prism version 8.0.

\section{Results}

Toothbrush data was collected from participants aged 12-50+ years. The frequency of toothbrush donation for each age category is as follows: 1217 years $(n=6), 18-22$ years $(n=32), 23-29$ years $(n=11), 30-39$ years $(\mathrm{n}=16), 40-49$ years $(\mathrm{n}=11)$ and $50+(\mathrm{n}=27)$. Analysis of agar plates using standard laboratory tests including agar and biochemical properties revealed that $97 \%$ of used toothbrushes were contaminated and the presence of the following organisms was observed; Enterobacteriaceae comprising Escherichia coli (62\%), Klebsiella species (55\%), Enterobacter species (4\%) and Serratia species (4\%), Staphylococcus species (70\%), non-fermenting Gram-negative bacteria (47\%) and Candida species (77\%). Further analysis included confirmation of the identity of Gram-negative organisms via MALDITOF MS. Results revealed the presence of Serratia liquifaciens, Enterobacter cloacae, Klebsiella oxytoca, Pseudomonas synxantha, Acinetobacter lwoffii, Acinetobacter johnsonii, Pantoea agglomerans and Pseudomonas fulva. The D'Agostino-Pearson normality test revealed that the microbial load data were not normally distributed. We, therefore, analysed differences in microbial loads using non-parametric tests. The microbial load numbers show median values of around $10^{7}$ cfu/brush head. Selective media show the median loads as approximately $10^{6}$ Candida/fungi per brush head and around $10^{5}$ of Enterobacteriaceae and Staphylococci. The microbial loads ranged from $0-10^{10} \mathrm{cfu} / \mathrm{brush}$ (Figure 1).

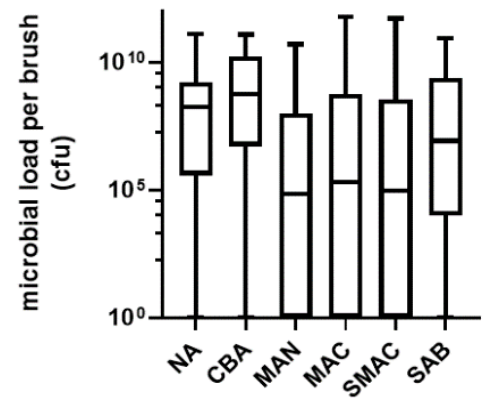

$p$ values:

NA vs MAN $<0.0001$

NA vs MAC $\quad 0.0004$

NA vs SMAC $\quad 0.0002$

CBA vs MAN $<0.0001$

CBA vs MAC $\quad 0.0014$

CBA vs SMAC $<0.0001$

MAN vs SAB $<0.0001$

SMAC VS SAB $\quad 0.0377$

Figure 1: Microbial populations (colony forming units, cfu/brush) following aerobic culture of used toothbrushes on different media. Media (\% from total of 103 of used toothbrushes showing growth): NA, nutrient agar (96\%); CBA, Columbia blood agar (97\%); MAN, mannitol salt agar (70\%); MAC, MacConkey agar (70\%); SMAC, Sorbitol MacConkey agar 65\%); SAB, Sabouraud dextrose agar 80\%). The graphs show the 25th to 75 th percentile range of microbial populations (boxes), the median (bars), and the maximum and minimum values (whiskers). $\mathrm{P}$ values show significant differences between the medians of the microbial populations on different media calculated by the Wilcoxon matched-pairs signed-rank test.

The following variables independently showed no significant differences in microbial levels on any media: gender, number of times per day brushing, toothbrush age, smoking, diet, toothpaste brand. Multiple linear regression did not identify combinations of factors that predicted the numbers of viable organisms found on toothbrushes. Although there was no difference between microbial levels, we noted that $90 \%$ of toothbrushes were stored in bathrooms presumably exposed to environmental contamination; 67 (72\%) contained Enterobacteriaceae, $72(77 \%)$ contained Staphylococci and $80(86 \%)$ contained Candida sp. We also noted that only $7 \%$ of participants used a protective cap and only 1 participant cleaned their toothbrushes after use. Amongst other factors 
that might have influenced microbial contamination, mouthwash was used by $50 \%$ of toothbrush donors and antibiotics had been received by $70 \%$ in the previous three months.

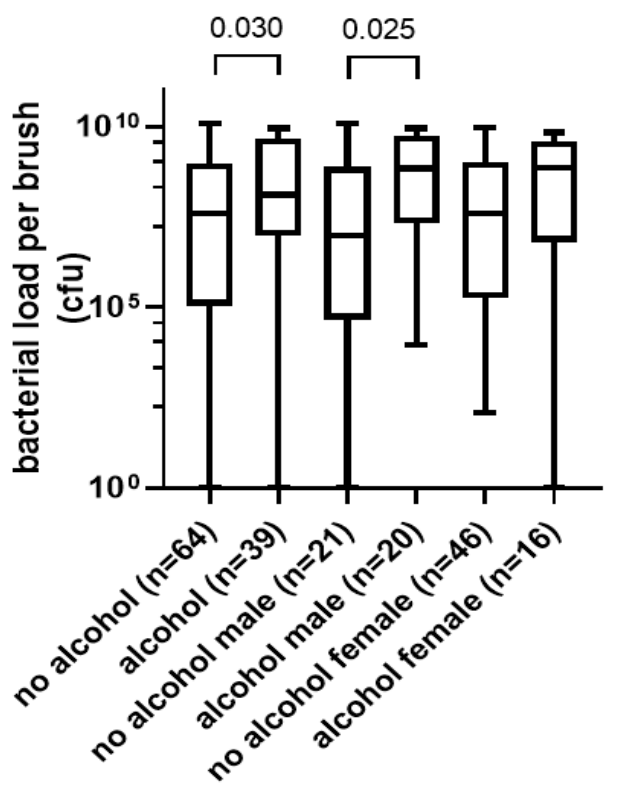

Figure 2: Effect of alcohol consumption on microbial loads isolated from CBA agar. The graphs show the 25th to 75th percentile range of microbial populations (boxes), the median (bars), and the maximum and minimum values (whiskers). $\mathrm{P}$ values show significant differences between the medians of the microbial populations calculated by the Mann-Whitney test.

\section{Effect of Alcohol Consumption by Donors on Toothbrush Contamination}

Participants were asked if they consumed alcohol. We analysed results stratifying brush donors as non-alcohol consumers or consumers (frequency of use defined as at least once a week). Investigating correlations between microbiological findings and questionnaire results showed that alcohol consumption had a significant influence upon the numbers of organisms cultured on CBA (Figure 2). Alcohol consumption by toothbrush donors revealed higher counts on CBA $(p=0.030)$. Further analysis showed that this significance was associated with males only ( $\mathrm{p}=0.025)$. Similar results were shown on NA (data not shown).

\section{Effect of Donors' Age on the Nature and Contamination Load Isolated from Toothbrushes}

The age group of brush donors had a significant influence upon the nature and numbers of organisms cultured on MAC, MAN and SAB (Figures 3a-3c). Significant differences were noted for ages 18-22 and $40-49$ years $(\mathrm{p}=0.035)$ and $30-39$ and $40-49$ years $(\mathrm{p}=0.033)$. The presence of Staphylococci was observed on MAN, results revealed significant differences between 23-29 and 30-39 years $(\mathrm{p}=0.024)$ and age groups $30-39$ and $40-40$ years $(\mathrm{p}=0.015)$. Presence of fungi and yeast was determined via $\mathrm{SAB}$ and multiple significant differences were noted as shown $(\mathrm{p} \leq 0.023)$. a

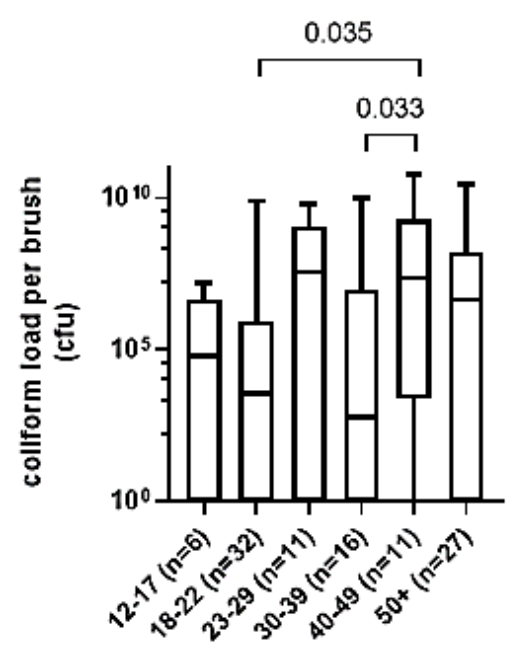

b

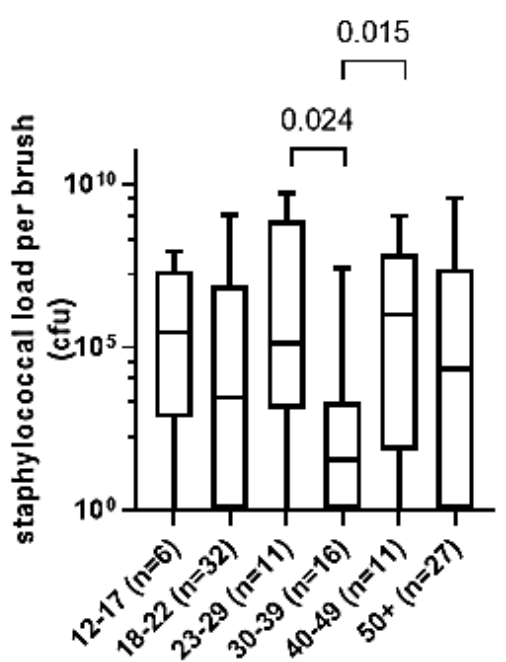

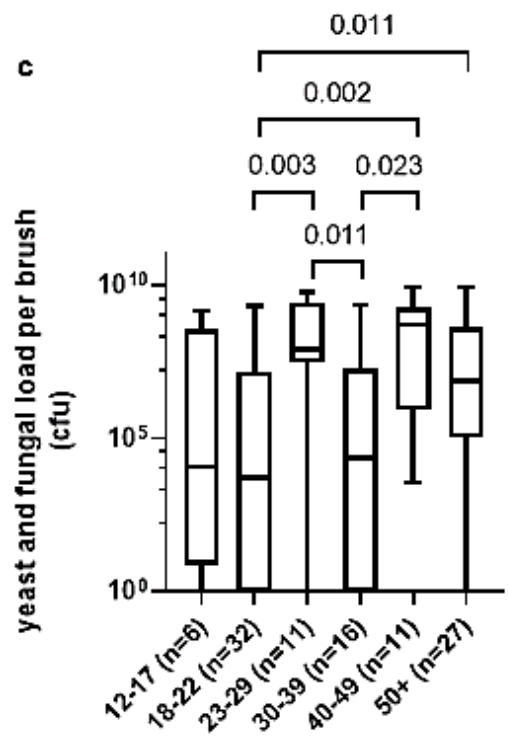

Figure 3: Microbial populations (colony forming units, cfu/brush) following aerobic culture of used toothbrushes on a) MAC, b) MAN and c) SAB. The graphs show the 25th to 75 th percentile range of microbial populations (boxes), the median (bars), and the maximum and minimum values (whiskers). Varying levels of Enterobacteriaceae were observed on MAC for toothbrushes across the age groups. Mann-Whitney tests were conducted to highlight any significant differences between median microbial loads on toothbrushes from different age groups.

\section{Effect of Toothbrush Age on the Nature and Contamination Load Observed on Different Media}

( $p>0.05)$. This suggests that maximum load was generated during the first three months of use and did not increase thereafter.

Figure 4 reveals that there was no significant difference between ages of brushes from 0-3 months to $>12$ months across all the agar types 

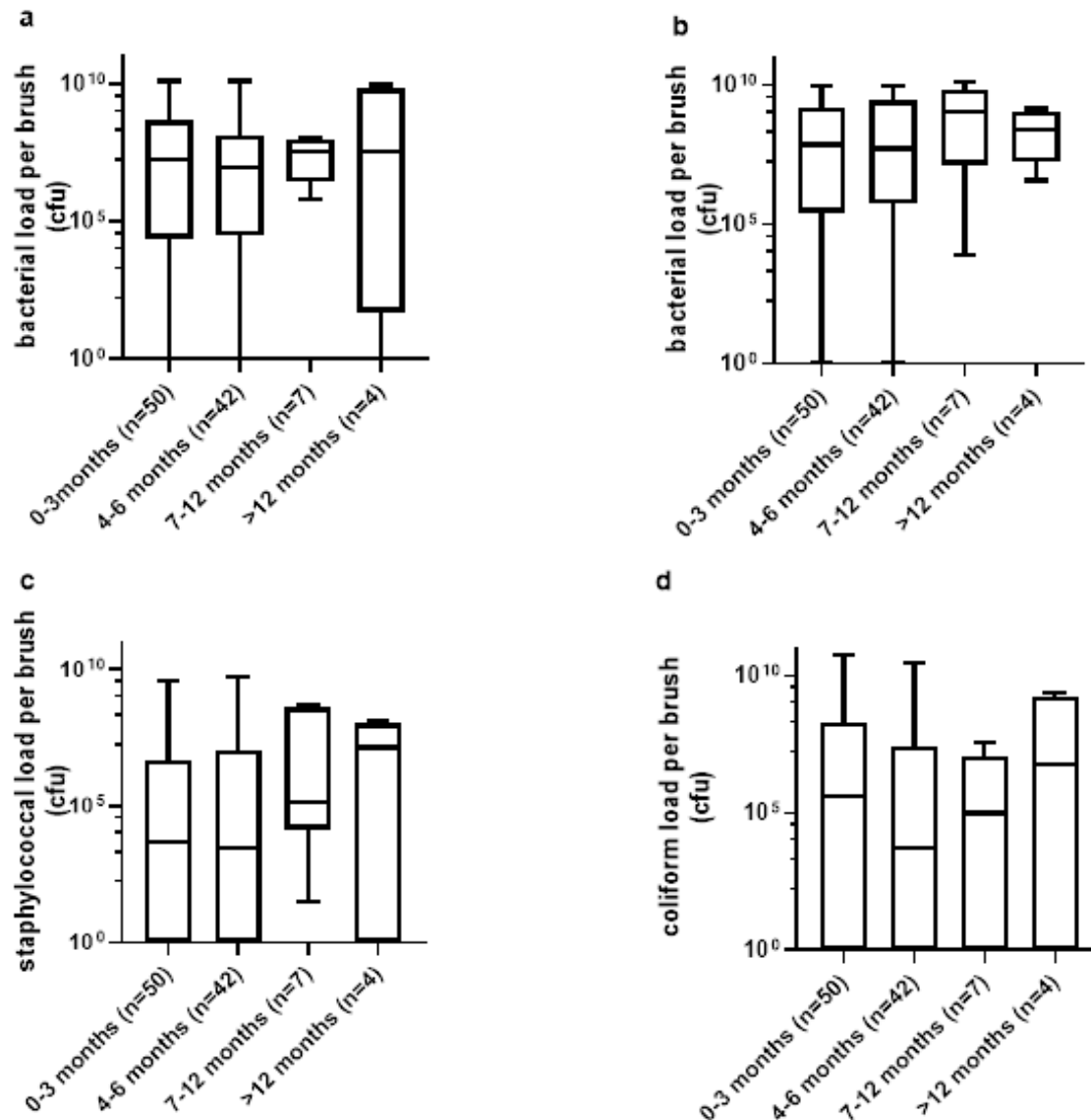

d
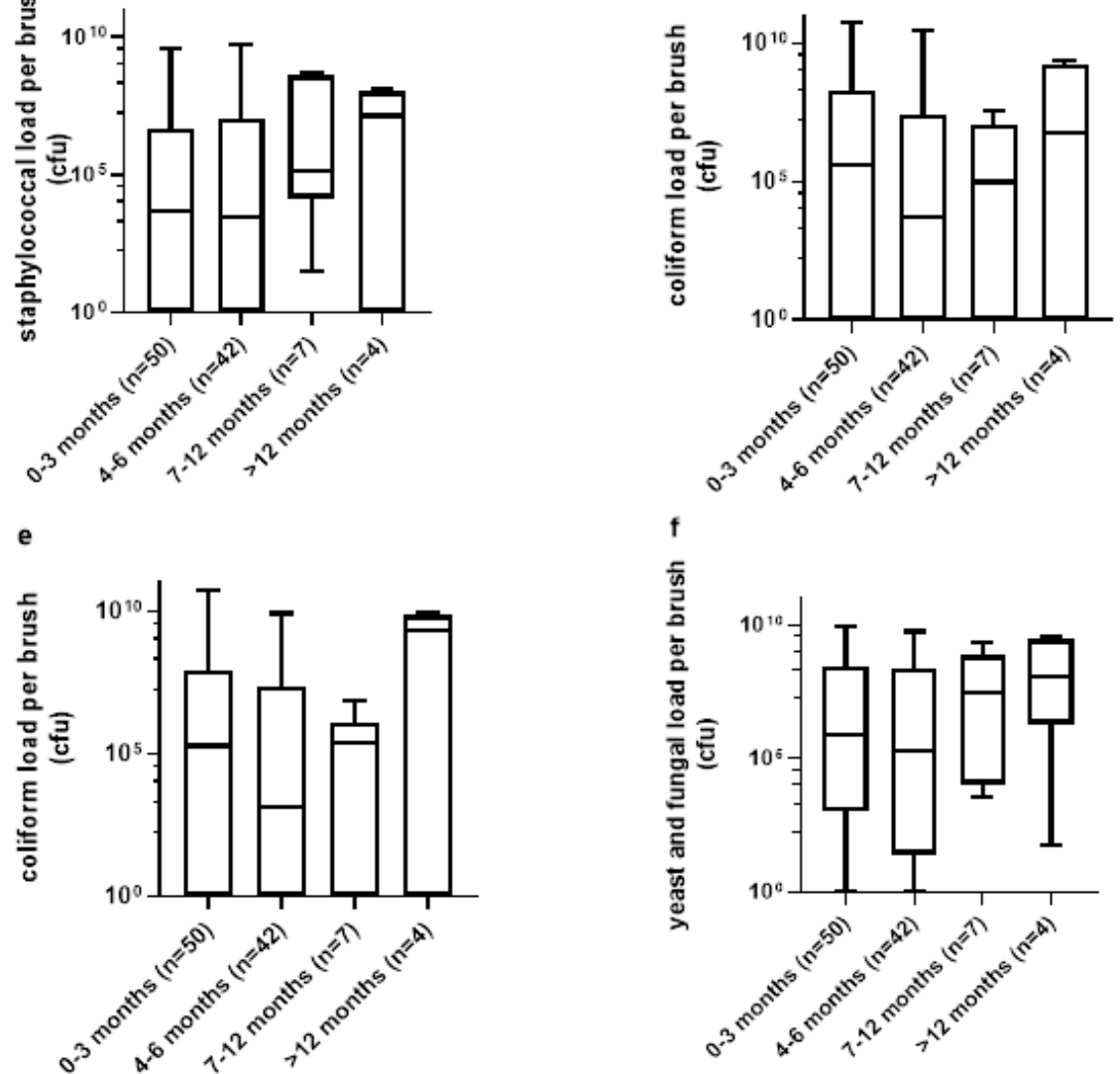

Figure 4: Microbial populations of used toothbrushes of different ages (colony forming units, cfu/brush) following aerobic culture on a) NA b) CBA and c) MAN d) MAC e) SMAC f) SAB. The graphs show the 25th to 75th percentile range of microbial populations (boxes), the median (bars), and the maximum and minimum values (whiskers). No significant differences between toothbrush age and microbial loads were observed by Mann-Whitney tests on each media.

\section{Discussion}

Previous research has identified common microorganisms found in the mouth and on toothbrushes. However, little research has been conducted to determine the effect of lifestyle and storage on toothbrush contamination. Thus, the aim of this study was to quantify and identify environmental microorganisms on used toothbrushes. A total of $97 \%$ of used toothbrushes were contaminated. This suggests that mouth-totoothbrush cross-contamination or environmental contamination may be occurring, and these microorganisms are able to survive and proliferate on toothbrushes. In concurrence with these results, previous findings have also reported high $(>70 \%)$ levels of contamination and similar nature of pathogenic organisms [7, 9, 13, 14].

Candida sp. were isolated in the highest density, low levels of these opportunistic pathogens are naturally found in the mucous membranes of the mouth and digestive tract, and introduction from the brush into the oral cavity during repeated use may lead to cross-contamination and 
subsequent candidiasis [15]. A pilot study identified Candida contamination in $70 \%$ of toothbrushes [8]. It is possible, due to the presence of other gut microorganisms that some Candida identified may have originated from the gut. Although not observed in our study, previous work has shown that cigarette smoke can cause Candida albicans growth and biofilm formation [11]. Furthermore, Candida infections are common in individuals following a course of antibiotics due to changes in the natural gut and mouth microbiome [16]. 90\% of toothbrushes were stored in bathrooms presumably exposed to environmental contamination and $67(72 \%)$ of these revealed the presence of Enterobacteriaceae. Previous research has reported that bacteria can travel through aerosolization up to 10 feet from a flushing toilet and air microorganism levels are highest following flushing a toilet [17]. Many premises are now designed with internal bathrooms with no windows, thus increasing the dissemination of microorganisms through air circulation. Some toothbrushes revealed Enterobacteriaceae levels ranging from $10^{5}$ to $10^{10}$; this is concerning as the introduction of high levels of these organisms poses a significant risk of cross-contamination and potential infection as the infectious dose of some Enterobacteriaceae is as little as $10 \mathrm{cfu}$ [18].

Lee and Lee used Illumina sequencing to identify microorganisms present on 10 toothbrushes from five donors. Each donor stored one toothbrush in the bathroom and one toothbrush in an office environment [19]. They reported the presence of; Enterococcus, Acinetobacter, Staphylococcus, Pseudomonas, Pantoea, Klebsiella, Lactobacillus, Streptococcus and Neisseria. Our study tested 103 toothbrushes and results also revealed the presence of both Gram-positive and Gramnegative organisms. However, the experimental approach was designed to detect and quantify the most numerous organisms rather than the diversity on each toothbrush. MALDI-TOF MS analysis confirmed the presence of a range of Enterobacteriaceae and Pseudomonas sp., including those reported by Lee and Lee [19]. These organisms are environmental and opportunistic pathogens, posing a risk, particularly to immunocompromised individuals [20-22].

In studying factors influencing microbial loads, we found that toothbrushes donated by male alcohol consumers had significantly higher levels of growth on CBA than brushes donated by non-drinkers. Although we found a difference in the numbers in male alcohol consumers, we did not find a significant difference in the types of organisms isolated from the toothbrushes. There are limited studies linking alcohol consumption and toothbrush contaminants; other work has studied the oral microbiome by sampling mouth washings of alcohol consumers. Fan et al. suggest that a decrease in some bacteria such as Lactobacilli in the oral microbiome of alcohol consumers results in an increase in alkaline-tolerant bacteria [10].

Changing toothbrushes within 3-4 months is a recommended practice worldwide and UK guidance from Public Health England (PHE) also states that brushes should have a small head with texture bristles and be changed every one to three months $[2,23]$. Our results showed that $50 \%$ of participants kept their toothbrushes for longer than three months and $40 \%$ retained toothbrushes for 4-6 months. This is in line with findings reported by Mansoori et al. [7]. Our findings revealed that microbial loads on toothbrushes are fully established during the first three months of use and do not increase further with prolonged use of up to 12 months. We did not measure the increase in microbial load over the first three months of use. However, a microbial load of $10^{5}-10^{10}$ organisms had established across the different media in this period; these included coliforms. Other research has reported a similar level of contamination on toothbrushes used for one month and three months [24]. Statistics show that $23 \%$ of 5 -year-old children and $31 \%$ of adults in England in 2019 have had dental decay and $25 \%$ of adults do not brush with a fluoride toothpaste twice a day [25]. Our results also revealed that only $57 \%$ of adult participants brushed their teeth twice a day and $41 \%$ brushed only once a day. Similarly, Mansoori et al. reported $62 \%$ of participants brushed twice a day [7]. This suggests that guidance on toothbrush age and brushing frequency is not being followed and further education and awareness is required. A limitation of the study is that we did not attempt to culture oral organisms including strict anaerobes, as we were interested in environmental contaminants.

Despite recognition of toilet aerosolization, this study has highlighted toothbrushes are still being stored in bathrooms without caps and there are high levels of potentially pathogenic contaminants on toothbrushes including Enterobacteriaceae and Pseudomonas sp., which may lead to serious infections. Recognition of the potential risk of infection from contaminated toothbrushes is of particular importance to immunocompromised individuals. Toothbrushes may act as a source of cross-contamination/infection, especially when multiple users store brushes in close contact. Whilst regular replacement of toothbrushes may help, more attention should be given to their storage and cleaning to minimise and control microbial loads. Although there is an abundance of available guidelines and advertisements in relation to toothbrush use, it is clear that more measures to improve public awareness are required.

\section{Clinical Relevance}

\section{Scientific Rationale for Study}

It is recognised that toothbrushes become contaminated with microorganisms in use and during storage.

\section{Principal Findings}

High levels of microbial contamination were observed on used toothbrushes. $90 \%$ of toothbrushes were stored in bathrooms presumably exposed to environmental contamination and $72 \%$ revealed the presence of enteric bacteria. $50 \%$ of participants kept toothbrushes for $>3$ months; microbial loads were fully established during the first three months of use and did not increase further with prolonged use.

\section{Practical Implications}

Guidance on toothbrush age, brushing frequency and storage is not being followed. Further education is required to improve public awareness.

\section{Acknowledgement}

We would like to thank the following individuals who contributed to the acquisition of the data; Anna Kwafo, Jaspreet Dandha, Jerene Chowdhury, Lauren Shaw, Maria Javed, Safiya Rajabali. We would also like to thank the Microbiology Department at Birmingham Children's Hospital for identification of isolates on the MALDI-TOF MS. 


\section{Author Contributions}

The authors jointly planned the study, analysed the data and wrote the manuscript. The individuals listed under acknowledgements carried out the microbial culture work.

\section{Ethical Approval}

The Aston University Ethics Committee (application number 1572) granted ethical approval.

\section{Conflicts of Interest}

None.

\section{REFERENCES}

1. American Dental Association (2019) Toothbrushes.

2. Public Health England (2014) Delivering better oral health: an evidence-based toolkit for prevention.

3. Frazelle MR, Munro CL (2012) Toothbrush Contamination: A Review of the Literature. Nursing Res Pract 2012.

4. Dewhirst FE, Chen T, Izard J, Paster BJ, Tanner ACR et al. (2010) The human oral microbiome. J Bacteriol 192: 5002-5017. [Crossref]

5. Lu M, Xuan S, Wang Z (2019) Oral microbiota: A new view of body health. Food Sci Hum Well 8: 8-15.

6. Karasneh JA, Al Habashneh RA, Marzouka NAS, Thornhill MH (2017) Effect of cigarette smoking on subgingival bacteria in healthy subjects and patients with chronic periodontitis. BMC Oral Health 17: 64. [Crossref]

7. Mansoori N, Bakar I, Shahid N, Syed M (2018) A survey of microbial contamination of toothbrushes among general population of Karachi. Prof Med J 25.

8. Taji SS, Rogers AH (2013) The microbial contamination of toothbrushes. A pilot study. Austral Dent J 43: 128-130.

9. Osho A, Thomas BT, Akande YA, Udor RD (2013) Toothbrushes as fomites. J Dent Or Hyg 5.

10. Fan X, Peters BA, Jacobs EJ, Gapstur SM, Purdue MP et al. (2018) Drinking alcohol is associated with variation in the human oral microbiome in a large study of American adults. Microbiome 6: 59. [Crossref]

11. Baboni FB, Barp D, de Azevedo Izidoro ACS, Samaranayake LP, Rosa EAR (2009) Enhancement of Candida albicans virulence after exposition to cigarette mainstream smoke. Mycopathologia 168: 227 235. [Crossref]

12. Kato I, Vasquez A, Moyerbrailean G, Land S, Djuric Z et al. (2017) Nutritional Correlates of Human Oral Microbiome. J Am Coll Nutr 36: 88-98. [Crossref]

13. Ferreira CA, Savi GD, Panatto AP, Generoso J, Barichello T (2012) Microbiological evaluation of bristles of frequently used toothbrushes. Dental Press J Orthod 17: 72-76.

14. Pesevska S, Ivanovski K, Mindova S, Kaftandzieva A, Ristoska S et al. (2016) Bacterial contamination of the toothbrushes. J Int Dent Med Res 9: 6-12.

15. Erdogan A, Rao SSC (2015) Small intestinal fungal overgrowth. Curr Gastroenterol Rep 17: 16. [Crossref]

16. Darwazeh AMG, Darwazeh TA (2014) What Makes Oral Candidiasis Recurrent Infection? A Clinical View. J Mycol 2014.

17. Barker J, Jones MV (2005) The potential spread of infection caused by aerosol contamination of surfaces after flushing a domestic toilet. $J$ Appl Microbiol 99: 339-347. [Crossref]

18. Food and Drug Administration (2012) Bad Bug Book; Handbook of foodborne Pathogenic Microorganisms and Natural Toxins. Pathogenic Escherichia coli Group. Second Edition.

19. Lee SY, Lee SY (2019) Assessment of bacterial contamination of toothbrushes using Illumina MiSeq. Oral Biol Res 43: 180-188.

20. Gershman MD, Kennedy DJ, Noble Wang J, Kim C, Gullion J et al. (2008) Multistate outbreak of Pseudomonas fluorescens bloodstream infection after exposure to contaminated heparinized saline flush prepared by a compounding pharmacy. Clin Infect Dis 47: 1372-1379. [Crossref]

21. Ku SC, Hsueh PR, Yang PC, Luh KT (2000) Clinical and microbiological characteristics of bacteremia caused by Acinetobacter 1woffii. Eur J Clin Microbiol Infect Dis 19: 501-505. [Crossref]

22. Leitner E, Zarfel G, Luxner J, Herzog K, Pekard Amenitsch S et al. (2015) Contaminated handwashing sinks as the source of a clonal outbreak of KPC-2-producing Klebsiella oxytoca on a hematology ward. Antimicrob Agents Chemother 59: 714-716. [Crossref]

23. Centers for Disease Control and Prevention (2016) Use \& Handling of Toothbrushes.

24. Raiyani CM, Arora R, Bhayya DP, Dogra S, Katageri AA et al. (2015) Assessment of microbial contamination on twice a day used toothbrush head after 1-month and 3 months: An in vitro study. J Nat Sci Biol Med 6: S44-S48. [Crossref]

25. Oral Health Foundation (2020) Oral health statistics in the UK. 